\title{
LOCALLY PRIME ARCS WITH FINITE PENETRATION INDEX
}

\section{BY J. MCPHERSON AND GEOFFREY HEMION}

Communicated by O. G. Harrold, September 29, 1973

Introduction. Let $k$ be an oriented arc in $R^{3}$, which has an isolated wild point $p$ at which the penetration index $P$ of $k$ is finite and no less than three. We say that $k$ is locally prime at $p$ if there is a tame closed 3-cell neighbourhood $U$ of $p$ which meets $k$ on its boundary in exactly $P$ points, such that if $\Gamma$ is any cube in Int $U$ which meets $k$ on its boundary in two points, then it is impossible for $k$ to be knotted in $\Gamma$; that is, $\pi_{1}(\Gamma-k)$ must be free cyclic. For example, the arc shown in Figure 2 of [2] is not locally prime at its wild endpoint, whereas the $\operatorname{arcs} A_{1}, A_{2}, \cdots$ of [1] are all locally prime at their respective endpoints.

The purpose of this paper is to announce the existence of a factorisation theorem for arcs which are not locally prime at their isolated wild points, to the effect that each nonlocally-prime arc can be decomposed into the "product" of a locally prime arc with a sequence of tame knots, and that this decomposition is unique; this extends the 1961 result of Fox and Harrold [5]. The proofs, which rely heavily on (sometimes tortuous) cutting and pasting arguments, will appear in another paper.

The second author is presently working on a more general factorisation theorem for arcs with isolated wild points and finite penetration index, to the effect that any such arc may be written as a finite "product" of arcs, so that each term in the product is an arc which is not itself the composite of two other wild arcs.

We have not yet explored the possibility of a more general theorem for arcs which are locally knotted in the sense of [9].

Results. Except in Theorem 1, we assume that our knots and arcs have only one wild point; the generalisation to knots and arcs with finitely many wild points is easily accomplished.

A cube is any tame set homeomorphic to $I^{3}$; it is well-placed (with respect to $k$ ) if it meets $k$ on its boundary in exactly two points. If $\Gamma$ is a wellplaced cube, we say that $k$ represents the knot $\kappa$ in $\Gamma$ if joining the endpoints of $k \cap \Gamma$ by a simple arc on $\mathrm{Bd} \Gamma$ yields an oriented knot equivalent to $\kappa$ in $R^{3}$, and $\Gamma$ is a $k$-prime cube if $k$ represents a (nontrivial) prime knot in $\Gamma$. We use the following fact from [10]:

LEMMA A. If $k$ represents the knot $\pi_{1} \# \cdots \# \pi_{m}$ in $\Gamma$ (where each $\pi_{i}$ is an oriented tame prime knot, and \# denotes tame knot multiplication),

AMS (MOS) subject classifications (1970). Primary 55A30. 
there exist $m$ disjoint well-placed cubes in Int $\Gamma$, say $\Gamma_{1}, \cdots, \Gamma_{m}$, such that $k$ represents $\pi_{i}$ in $\Gamma_{i}$, for each $i$.

We use the word " 3 -cell" in a restricted sense, in that a cube is a 3-cell only when it is a neighborhood of $p$ and meets $k$ on its boundary in exactly $P$ points; we also assume that our 3-cells are "small enough", in that if $E$ is a 3-cell and $C$ a cube which is a neighborhood of $p$, then $\mathrm{Bd} C$ contains at least $P$ points of $k$. An admissible sequence of 3-cells for $k$ is a sequence $E_{0} \supset E_{1} \supset \cdots$ of 3-cells for which $E_{i+1} \subset$ Int $E_{i}$ and $\cap E_{i}=p$.

The following lemmas are crucial; in each, $E_{0} \supset E_{1} \supset \cdots$ is an admissible sequence for $k$, and our well-placed cubes are assumed to lie in Int $E_{0}$.

LEMMA B. The fundamental group homomorphisms induced by the inclusions of $\mathrm{Bd} E_{\imath}-k$ in $E_{\imath}-k$-Int $E_{i+1}, E_{0}-k-\operatorname{Int} E_{i}$ and $E_{i}-k$, and of $\mathrm{Bd} E_{i+1}-k$ in $E_{i}-k$-Int $E_{i+1}$, are monomorphisms, $i \geqq 0$.

Lemma C. Let $\Gamma$ be a k-prime cube. There exists a well-placed cube $\Gamma^{*}$ in $\operatorname{Int}\left(E_{i}-E_{i+1}\right)$, some $i$, in which $k$ represents the same prime as it does in $\Gamma$. Moreover, we may assume that $\Gamma_{1}^{*}$ and $\Gamma_{2}^{*}$ are disjoint if $\Gamma_{1}$ and $\Gamma_{2}$ are disjoint $k$-prime cubes.

LEMMA D. We fix an index $i$ and work in $\operatorname{Int}\left(E_{i}-E_{i+1}\right)$. Let $\Gamma_{1}, \cdots, \Gamma_{n}$ be a family of disjoint well-placed cubes, and let $\Gamma$ be a k-prime cube. There exists a well-placed cube $\Gamma^{*}$ in which $k$ represents the same prime as in $\Gamma$, and such that $\mathrm{Bd} \Gamma^{*}$ meets none of the surfaces $\mathrm{Bd} \Gamma_{1}, \cdots, \mathrm{Bd} \Gamma_{n}$. Moreover, we may assume that $\Gamma_{a}^{*}$ and $\Gamma_{b}^{*}$ are disjoint if $\Gamma_{a}$ and $\Gamma_{b}$ are disjoint $k$-prime cubes.

One consequence of Lemmas $\mathrm{B}$ and $\mathrm{C}$ is the following: If $\pi_{1}\left(E_{i}-E_{i+1}-k\right)$ is free for each $i$, then there are no $k$-prime cubes in Int $E_{0}$ (for the existence of a $k$-prime cube in Int $E_{0}$ implies that $\pi_{1}\left(E_{i}-E_{i+1}-k\right)$ has a nonfree knot group as a subgroup, for some $\left.i\right)$.

Another consequence of Lemmas B and C, and the finiteness theorem of [6, p. 48], is that there are only finitely many disjoint $k$-prime cubes in $\operatorname{Int}\left(E_{i}-E_{i+1}\right)$, for any $i$. Note that this result is not true for tame arcs, but [5] shows it is true for Wilder arcs.

DEFINITION. Let $\kappa$ be an infinite sequence of (nontrivial) oriented prime tame knots, and $k_{0}$ an arc which has finite penetration index at its single wild point $p_{0}$. The arc $k_{1}$ with wild point $p_{1}$ (with $P\left(k_{1}, p_{1}\right)=$ $\left.P\left(k_{0}, p_{0}\right) \geqq 3\right)$ is (locally) the product of $k_{0}$ with the sequence $\kappa$, written $k_{1}=k_{0} \# \kappa$, if there exist infinitely many disjoint $k_{1}$-prime cubes $\Gamma_{i}$ converging to $p_{1}$, such that

(i) the prime knot types represented by $k_{1}$ in this sequence of cubes are in one-one onto correspondence with the prime knots of the sequence $\kappa$, and

(ii) if $\gamma_{i}$ is an oriented arc running from the starting point of $k_{1} \cap \Gamma_{i}$ 
along $\mathrm{Bd} \Gamma_{i}$ to the endpoint of $k_{1} \cap \Gamma_{i}$, then the consistently oriented arc $\bigcup\left(\gamma_{i} \cup\left(k_{1}-\Gamma_{i}\right)\right)$ has the same oriented local type at $p_{1}$ as $k_{0}$ has at $p_{0}$. Figure 2 of [2] shows the (nonoriented) product of Example 1.2 of [4] with an infinite sequence of trefoil knots.

Note that if $p_{0}$ is an interior point of $k_{0}$, and $\kappa^{*}$ the Wilder arc formed from the sequence $\kappa$, then $k_{0} \# \kappa$ is the interior composite $k_{0} \$_{p} \kappa^{*}$ (cf. [7, V.C. 7]).

Definition. The arc $k$ is locally prime at $p$ if it cannot be expressed as the product of another arc with an infinite sequence of nontrivial oriented prime knots.

An arc which is not locally prime at an interior wild point is locally knotted at that point [9].

THEOREM 1. The following arcs are locally prime at their respective wild points:

(i) Examples 1.1 and $1.1^{*}$ of [4],

(ii) The $\operatorname{arcs} A_{1}, A_{2}, \cdots$ of $[\mathbf{1}]$,

(iii) Example 5 of [3],

(iv) The arc $A_{0}$ of $[8]$.

For, each of these arcs has an admissible sequence for which $\pi_{1}\left(E_{i}-E_{i+1}-k\right)$ is free. For (iv), for example, the section $A_{0}, E_{i}, E_{i+1}$ is shown in the figure. Using the relations given in [8], it is straightforward to

$\mathrm{Bd} E_{i}$

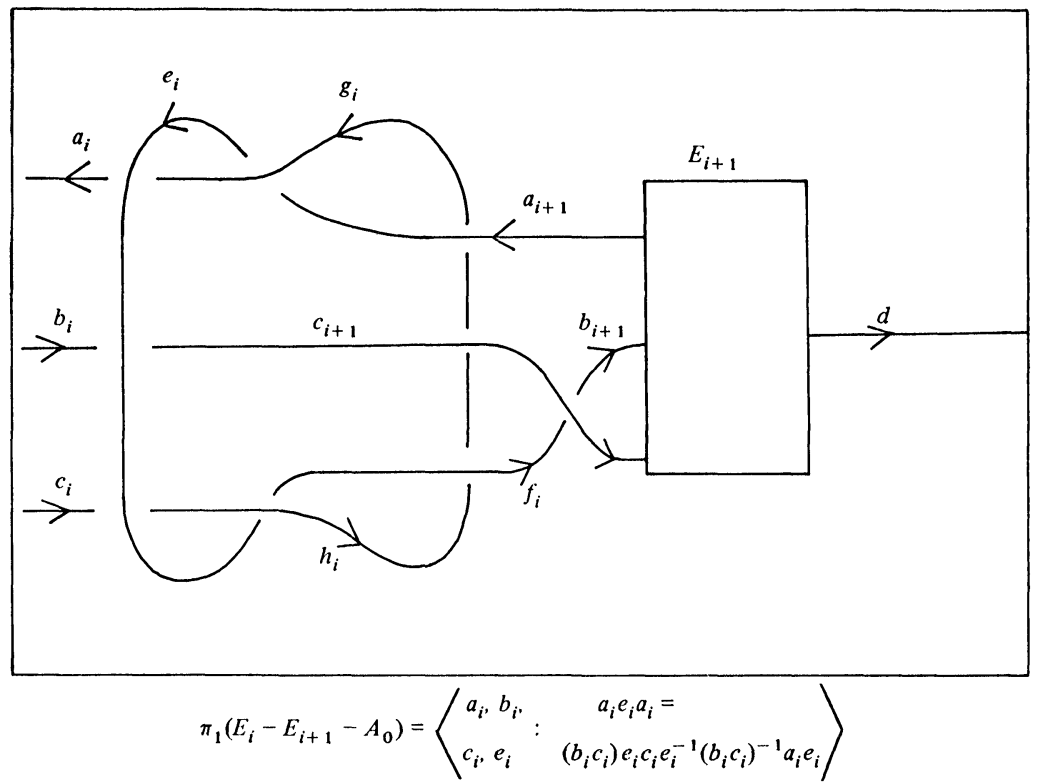


show that the fundamental group is the free group generated by the set $\left\{e_{i}, a_{i}, b_{i} c_{i}\right\}$.

THEOREM 2. Let $k$ be an arc which is not locally prime at $p$. Then there exists an arc $k^{*}$ which is locally prime at $p$, and a sequence $\kappa$ of prime knots, such that $k=k^{*} \# \kappa$. That is, every arc which is not locally prime has a prime factorisation in Int $E_{0}$.

Let $\pi_{1}, \pi_{2}, \cdots$ be an ordering of the oriented prime tame knots, and let $\kappa$ be a sequence of such knots. For each $i$, let $e_{i}(\kappa)$ denote the number of times the prime $\pi_{i}$ occurs in $\kappa$; if $\pi_{i}$ occurs infinitely often, set $e_{i}(\kappa)=\infty$.

THEOREM 3. Let $k$ be an arc which is not locally prime at its wild point, and let $k_{1} \# \kappa_{1}$ and $k_{2} \# \kappa_{2}$ be prime factorisations of $k$. Then $k_{1}$ and $k_{2}$ are locally equivalent, and

(i) $e_{i}\left(\kappa_{2}\right)=\infty$ iff $e_{i}\left(\kappa_{1}\right)=\infty$, for a given $i$; that is, $\kappa_{1}$ and $\kappa_{2}$ have the same set of infinitely occurring primes, and

(ii) if $e_{i}\left(\kappa_{1}\right)$ is finite, then $e_{i}\left(\kappa_{2}\right)=e_{i}\left(\kappa_{1}\right)$; and this is true for all but finitely many $i$.

\section{REFERENCES}

1. W. R. Alford and B. J. Ball, Some almost polyhedral wild arcs, Duke Math. J. 30 (1963), 33-38. MR 26 \#1858.

2. B. J. Ball, Penetration indices and applications, Topology of 3-Manifolds and Related Topics (Proc. Univ. of Georgia Inst., 1961), Prentice-Hall, Englewood Cliffs, N.J., 1962, pp. 37-39. MR 25 \#4506.

3. R. H. Fox, On shape, Fund. Math. 74 (1972), no. 1, 47-71. MR 45 \#5973.

4. R. H. Fox and E. Artin, Some wild cells and spheres in three-dimensional space, Ann. of Math. (2) 49 (1948), 979-990. MR 10, 317.

5. R. H. Fox and O. G. Harrold, The Wilder arcs, Topology of 3-Manifolds and Related Topics (Proc. Univ. of Georgia Inst., 1961), Prentice-Hall, Englewood Cliffs, N.J., 1962, pp. 184-187. MR 25 \#3519.

6. W. Haken, Some results on surfaces in 3-manifolds, Studies in Modern Topology, Math. Assoc. Amer. (distributed by Prentice-Hall, Englewood Cliffs, N.J.), 1968, pp. 39-98. MR 36 \#7118.

7. S. J. Lomonaco, An algebraic theory of local knottedness. I, Trans. Amer. Math. Soc. 129 (1967), 322-343. MR 36 \#877.

8. - Uncountably many mildly wild non-Wilder arcs, Proc. Amer. Math. Soc. 19 (1968), 895-898. MR 37 \#2199.

9. G. G. Miller, Locally knotted one-dimensional manifolds in three-space, University of Victoria, Report No. 53.

10. H. Schubert, Die eindeutige Zerlegbarkeit eines Knotens in Primknoten, S.-B. Heidelberger Akad. Wiss. Math. Nat. Kl. 1949, no. 3, 57-104. MR 11, 196.

Department of Mathematics, Australian National University, Canberra, Australia 